\title{
Advances in IC-Scheduling Theory: Scheduling Expansive and Reductive Dags and Scheduling Dags via Duality
}

\author{
Gennaro Cordasco* \\ Univ. of Salerno
}

\author{
Grzegorz Malewicz \\ Google, Inc.
}

\author{
Arnold L. Rosenberg $\ddagger$ \\ Univ. of Massachusetts
}

\begin{abstract}
Earlier work has developed the underpinnings of IC-Scheduling Theory, a framework for scheduling computations having intertask dependencies - modeled via dagsfor Internet-based computing. The goal of the schedules produced is to render tasks eligible for execution at the maximum possible rate, with the dual aim of: $(a)$ utilizing remote clients' computational resources well, by always having work to allocate to an available client; $(b)$ lessening the likelihood of a computation's stalling for lack of eligible tasks. The dags handled by the Theory thus far are those that can be decomposed into a given collection of bipartite building-block dags via the operation of dag-decomposition. A basic tool in constructing schedules is a relation $\triangleright$, which allows one to "prioritize" the scheduling of a complex dag's building blocks. The current paper extends IC-Scheduling Theory in two ways: by expanding significantly the repertoire of dags that the Theory can schedule optimally, and by allowing one sometimes to shortcut the algorithmic process required to find optimal schedules. The expanded repertoire now allows the Theory to schedule optimally, among other dags, a large range of dags that are either "expansive," in the sense that they grow outward from their sources, or "reductive," in the sense that they grown inward toward their sinks. The algorithmic shortcuts allow one to "read off" an optimal schedule for a dag from a given optimal schedule for the dag's dual, which is obtained by reversing all arcs (thereby exchanging the roles of sources and sinks).
\end{abstract}

Index terms. IC-Scheduling theory, Internet-based computing, Grid computing, Global computing, Scheduling dags, Theory

\footnotetext{
${ }^{*}$ Dip. di Informatica e Applicazioni, Univ. di Salerno, Fisciano 84084, ITALY, cordasco@dia.unisa.it

${ }^{\dagger}$ Dept. of Engineering, Google Inc., Mountain View CA 94043, USA, malewicz@google.com

${ }^{\ddagger}$ Dept. of Computer Science, Univ. of Massachusetts, Amherst, MA 01003, USA, rsnbrg@cs .umass .edu
} 


\section{Introduction}

Earlier work $[16,18,19]$ has developed the underpinnings of $I C$-Scheduling Theory, a formal framework for studying the problem of scheduling computations having intertask dependencies for the several modalities of Internet-based computing (IC, for short) - including Grid computing (cf. [1, 8, 7]), global computing (cf. [2]), and Web computing (cf. [13]). The goal is to craft schedules that maximize the rate at which tasks are rendered eligible for allocation to (hence for execution by) remote computing workers (henceforth, clients), with the dual aim of: (a) enhancing the effective utilization of remote clients, by always having work to allocate to an available client; $(b)$ lessening the likelihood of a computation's stalling pending the completion of already-allocated tasks. The impetus for this new theory is temporal unpredictability: Communication with remote clients proceeds over the Internet, hence may experience unpredictable delays; remote clients are not dedicated to remotely allocated work, hence may execute that work at an unpredictable rate. These sources of unpredictability make it difficult to accurately identify critical paths in complex computations and, thereby, to successfully apply traditional scheduling techniques. Consequently, a new scheduling paradigm is needed, that acknowledges both the strengths and weaknesses of the Internet as a computational medium.

As a simple, but dramatic, illustration of the need to pay attention to the rate of rendering tasks eligible for execution, Fig. 1 depicts two strategies for scheduling wavefront computations (the second dag in Fig. 2). Under the lefthand, "diagonal-shells," strategy
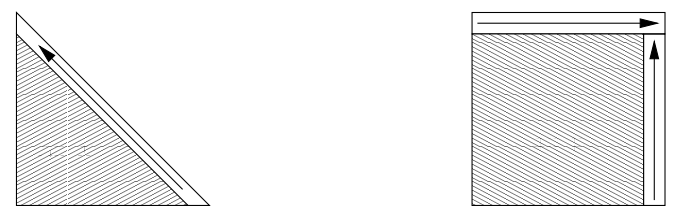

Figure 1: Scheduling wavefront computations by "diagonal" and "square" shells.

(which is optimal [18]), roughly $\sqrt{T}$ tasks are eligible after $T$ task-executions; under the righthand, "square-shells," strategy, there are never more than three eligible tasks. Thus, during periods when a large number of clients are available, the "diagonal-shells" schedule can fruitfully exploit many of them, while the "square-shells" schedule cannot.

The framework of $[16,18,19]$ idealizes the problem of scheduling computation-dags ${ }^{1}$ for IC, via the assumption that tasks are executed in the order of their allocation. (This assumption idealizes the hope that the monitoring of clients' past behaviors and current capabilities prescribed in, say, $[1,12,20]$ can render the desired order likely, if not certain.) Building on the case studies of $[18,19]$, in [16] we developed what we hope will be the

\footnotetext{
${ }^{1}$ All technical terms are defined in Section 2.2. For brevity, we henceforth speak of "dags," rather than "computation-dags."
} 
underpinnings of a theory of scheduling complex dags for IC. The development in [16] begins with any collection of building-block dags that we know how to schedule optimally. It develops two conceptual/algorithmic notions that allow one to schedule complex dags built from these building blocks, using a suite of algorithms that optimally schedule dags that arise from a large variety of significant computations.

1. The priority relation $\triangleright$ on pairs of dags. The assertion " $\mathcal{G}_{1} \triangleright \mathcal{G}_{2}$ " asserts that the schedule $\Sigma$ that entirely executes $\mathcal{G}_{1}$ and then entirely executes $\mathcal{G}_{2}$ is at least as good (relative to our quality metric) as any other schedule that executes both $\mathcal{G}_{1}$ and $\mathcal{G}_{2}$.

2. The operation of composition on pairs of dags, that builds up simple dags into complex ones.

The suite of algorithms developed in [16] decompose a given dag $\mathcal{G}$ into building blocks that compose to produce $\mathcal{G}$. One can often use $\triangleright$-priorities among $\mathcal{G}$ 's building blocks to compute an optimal schedule for $\mathcal{G}$ from optimal schedules for its building blocks.

While many dags do not admit optimal schedules under IC-Scheduling Theory, ${ }^{2}$ the dags that arise from a large variety of disparate, important computations can be scheduled optimally via the algorithms of [16]. Fig. 2 depicts four such dags whose optimal schedules
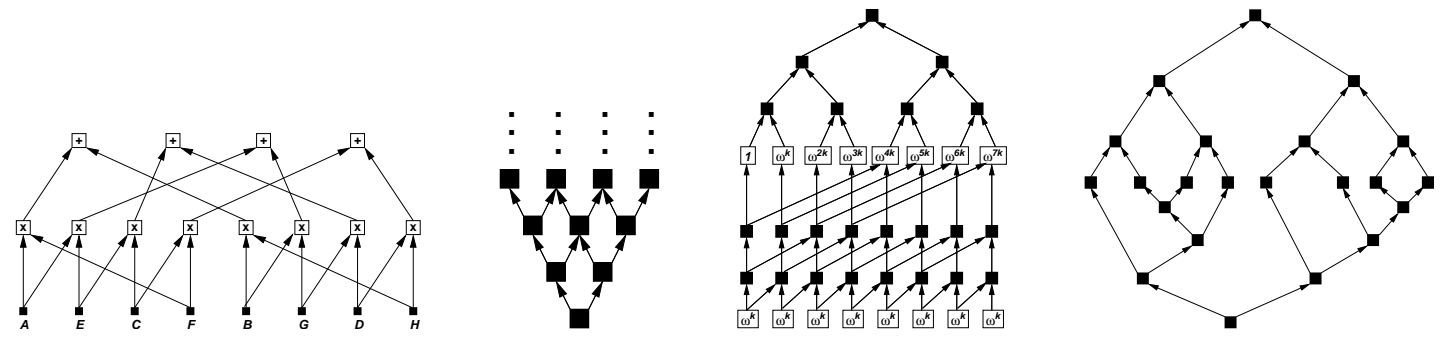

Figure 2: Data-dependency dags for four computations: matrix multiplication, a wavefront computation, the discrete Laplace transform, a divide-and-conquer computation.

are derived in [5], using the algorithmic framework of [16]. Techniques from the current paper greatly streamline the derivations of these optimal schedules. More importantly, the current paper extends the framework of [16] in two significant ways.

(1) In Section 3, we significantly augment the repertoire of building-block dags that the scheduling algorithms of [16] can accommodate when seeking optimal schedules. This augmentation allows the theory to schedule optimally, inter alia, a large range of dags that are either "expansive," in the sense that they grow outward from their sources, or "reductive," in the sense that they grown inward toward their sinks; some structurally

\footnotetext{
${ }^{2}$ Our optimal schedules must maximize the number of eligible nodes at every step of the computation, so many dags do not admit optimal schedules [16]. For such dags, the initial task-executions that maximize the number of eligible tasks at time $t_{1}$ differs from those that maximize the number at some $t_{2}>t_{1}$.
} 
uniform such dags are exemplified in Fig. 2. The algorithms can also now handle a large range of compositions of "expansive" dags with "reductive" dags, as exemplified in Fig. 3.
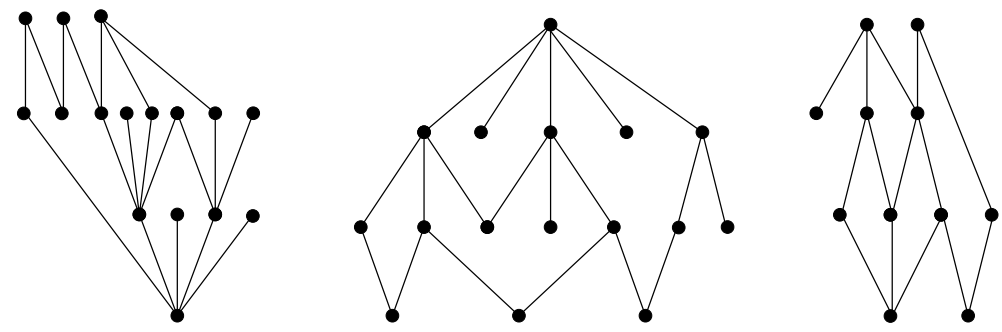

Figure 3: Three composite dags that our expanded framework can schedule optimally; all edges represent arcs that point upward. Only the middle of these dags could be scheduled optimally using the framework of [16].

(2) In Section 4, we show how to "read off" two algorithmically significant features of a $\operatorname{dag} \mathcal{G}$ from analogous features for $\mathcal{G}$ 's dual dag $\widetilde{\mathcal{G}}$ - which is obtained by reversing all of $\mathcal{G}$ 's arcs. (a) We show how to "read off" an optimal schedule for $\mathcal{G}$ from a given optimal schedule for $\widetilde{\mathcal{G}}$, if one exists (Section 4.1). One can, e.g., "read off" an optimal schedule for either an "expansive" or a "reductive" dag from a given optimal schedule for the other. (b) We show how to "read off" $\triangleright$-priorities among dags from known $\triangleright$-priorities among their duals (Section 4.2).

Thus, in this paper, we expand the repertoire of dags that the nascent theory of [16] can schedule optimally, and we provide tools that often allow one to avoid the low-degree polynomial, yet not-insignificant, suite of algorithms used in [16] to find optimal schedules.

Related work. Most closely related to our study are its companions in developing ICScheduling Theory: [16], whose contributions we have just described; [18, 19], case studies that characterize and specify optimal schedules for a variety of uniform dags; [4], wherein the algorithmic framework of [16] is extended in several ways; [5], wherein optimal algorithms are designed for a range of disparate computational problems, including those in Fig. 2 ; $[10,14]$, which evaluate the computational impact of our idealized scheduling theory via simulation experiments - and suggest that this impact is quite positive for a substantial range of plausible scenarios. A companion to these sources, [15]-which is motivated by the fact that many dags do not admit an optimal schedule in the sense of [16] - pursues an orthogonal regimen for scheduling dags for IC, in which a server allocates batches of tasks periodically, rather than allocating individual tasks as soon as they become eligible. Optimality is always possible within the batched framework, but achieving it may entail a prohibitively complex computation. In less directly related work, [9] presents a probabilistic approach to the problem of executing tasks on unreliable clients. Finally, the impetus for our study derives from the many exciting systems- and/or application-oriented studies of IC, in sources such as $[1,2,7,8,12,13,20]$. 


\section{A Basis for a Scheduling Theory}

\subsection{Computation-Dags}

A directed graph $\mathcal{G}$ is given by a set of nodes $N_{\mathcal{G}}$ and a set of arcs (or, directed edges) $A_{\mathcal{G}}$, each of the form $(u \rightarrow v)$, where $u, v \in N_{\mathcal{G}}$. A path in $\mathcal{G}$ is a sequence of arcs that share adjacent endpoints, as in the following path from node $u_{1}$ to node $u_{n}$ : $\left(u_{1} \rightarrow u_{2}\right),\left(u_{2} \rightarrow\right.$ $\left.u_{3}\right), \ldots,\left(u_{n-2} \rightarrow u_{n-1}\right),\left(u_{n-1} \rightarrow u_{n}\right)$. A dag (directed acyclic graph) $\mathcal{G}$ is a directed graph that has no cycles - so that no path of the preceding form has $u_{1}=u_{n}$. When a dag $\mathcal{G}$ is used to model a computation, i.e., is a computation-dag (henceforth, dag):

- each node $v \in N_{\mathcal{G}}$ represents a task in the computation;

- an $\operatorname{arc}(u \rightarrow v) \in A_{\mathcal{G}}$ represents the dependence of task $v$ on task $u$ : $v$ cannot be executed until $u$ is.

For any $\operatorname{arc}(u \rightarrow v) \in A_{\mathcal{G}}, u$ is a parent of $v$, and $v$ is a child of $u$ in $\mathcal{G}$. The indegree (resp., outdegree) of node $u$ is its number of parents (resp., children). A parentless node of $\mathcal{G}$ is a source, and a childless node is a sink. $\mathcal{G}$ is connected if, when one ignores the orientation of its arcs, there is a path connecting every pair of distinct nodes; $\mathcal{G}$ is bipartite if $N_{\mathcal{G}}$ consists entirely of sources and $\operatorname{sinks}^{3}$; we refer to a connected bipartite dag as a $C B B B$, for Connected Bipartite Building Block (a term whose origins will become clear later).

Let the dags $\mathcal{G}_{1}$ and $\mathcal{G}_{2}$ be disjoint, in the sense that $N_{\mathcal{G}_{1}} \cap N_{\mathcal{G}_{2}}=\emptyset$. The sum (or, disjoint union) of $\mathcal{G}_{1}$ and $\mathcal{G}_{2}$, denoted $\mathcal{G}_{1}+\mathcal{G}_{2}$, is the dag whose node-set is $N_{\mathcal{G}_{1}} \cup N_{\mathcal{G}_{2}}$ and whose arc-set is $A_{\mathcal{G}_{1}} \cup A_{\mathcal{G}_{2}}$.

\subsection{A Model for Executing Dags on the Internet}

"Pebble games" on dags have yielded elegant formalizations of a variety of problems related to scheduling dags. Such games use tokens, pebbles, to model the progress of a computation on a dag: the placement or removal of the various available types of pebbles - which is constrained by the dependencies modeled by the dag's arcs - represents the changing (computational) status of the dag's task-nodes. Our study is based on the Internet-Computing (IC, for short) Pebble Game of [18]. Based on studies of IC in, e.g., [1, 12, 20], arguments are presented in $[18,19]$ (q.v.) that justify the simplified form of the Game that we study.

A. The rules of the Game. The IC Pebble Game on a dag $\mathcal{G}$ involves one player $S$, the Server, who has access to unlimited supplies of two types of pebbles: ELIGIBLE pebbles, whose presence indicates a task's eligibility for execution, and EXECUTED pebbles, whose presence indicates a task's having been executed. The Game is played as follows.

\footnotetext{
${ }^{3}$ Perforce, all arcs go from a source to a sink.
} 
The IC Pebble Game

- $S$ begins by placing an ELIGIBLE pebble on each unpebbled source of $\mathcal{G}$.

/*Unexecuted sources are always eligible for execution, having no parents whose prior execution they depend on.*/

- At each step, $S$

- selects a node that contains an ELIGIBLE pebble,

- replaces that pebble by an EXECUTED pebble,

- places an ELIGIBLE pebble on each unpebbled node of $\mathcal{G}$ all of whose parents contain EXECUTED pebbles.

- $S$ 's goal is to allocate nodes in such a way that every node $v$ of $\mathcal{G}$ eventually contains an EXECUTED pebble.

/*This modest goal is necessitated by the possibility that $\mathcal{G}$ is infinite.*/

A schedule for the IC Pebble Game is a rule for selecting which ELIGIBLE pebble to execute at each step of a play of the Game. For brevity, we henceforth call a node ELIGIBLE (resp., EXECUTED) when it contains an ELIGIBLE (resp., an EXECUTED) pebble. For uniformity, we henceforth talk about executing nodes rather than tasks.

B. The quality of a play of the Game. Our goal is to play the IC Pebble Game in a way that maximizes the production rate of ELIGIBLE pebbles. When $\mathcal{G}$ is bipartite, it suffices to focus on maximizing the production rate of ELIGIBLE sinks. For each step $t$ of a play of the Game on a dag $\mathcal{G}$ under a schedule $\Sigma$, let $E_{\Sigma}(t)$ denote the number of ELIGIBLE pebbles on $\mathcal{G}$ 's nonsource nodes at step $t$.

We measure the $\mathbf{I C}$ quality of a play of the IC Pebble Game on a dag $\mathcal{G}$ by the size of $E_{\Sigma}(t)$ at each step $t$ of the play-the bigger, the better. Our goal is an IC-optimal schedule $\Sigma$, in which $E_{\Sigma}(t)$ is as big as possible for all steps $t$.

The significance of IC quality - hence of IC optimality - stems from the following scenarios. (1) Schedules that produce ELIGIBLE nodes more quickly may reduce the chance of the "gridlock" that could occur when remote clients are slow-so that new tasks cannot be allocated pending the return of already allocated ones. (2) If the IC Server receives a batch of requests for tasks at (roughly) the same time, then having more ELIGIBLE tasks available allows the Server to satisfy more requests.

While our scheduling model is very idealized, preliminary simulation experiments, in [14, 10] suggest that schedules with greater IC quality actually decrease computation completion times in a large range of Internet-based computing scenarios. 


\subsection{A Framework for Crafting IC-Optimal Schedules}

The priority relation $\triangleright$. For $i=1,2$, let the dag $\mathcal{G}_{i}$ have $n_{i}$ nonsinks, and let it admit the IC-optimal schedule $\Sigma_{i}$. If the following inequalities hold: ${ }^{4}$

$$
\begin{aligned}
& \left(\forall x \in\left[0, n_{1}\right]\right)\left(\forall y \in\left[0, n_{2}\right]\right): \\
& \quad E_{\Sigma_{1}}(x)+E_{\Sigma_{2}}(y) \leq E_{\Sigma_{1}}\left(\min \left\{n_{1}, x+y\right\}\right)+E_{\Sigma_{2}}\left(\max \left\{0, x+y-n_{1}\right\}\right),
\end{aligned}
$$

then $\mathcal{G}_{1}$ has priority over $\mathcal{G}_{2}$, denoted $\mathcal{G}_{1} \triangleright \mathcal{G}_{2}$. Informally, one never decreases IC quality by executing a nonsink of $\mathcal{G}_{1}$ whenever possible.

A framework for scheduling complex dags. The operation of composition is defined inductively as follows.

- Start with a set $\mathcal{B}$ of base dags. ${ }^{5}$

- One composes dags $\mathcal{G}_{1}, \mathcal{G}_{2} \in \mathcal{B}$ - which could be the same dag with nodes renamed to achieve disjointness - to obtain a composite dag $\mathcal{G}$, as follows.

- Let $\mathcal{G}$ begin as the sum $\mathcal{G}_{1}+\mathcal{G}_{2}$, with nodes renamed if necessary to ensure that $N_{\mathcal{G}} \cap\left(N_{\mathcal{G}_{1}} \cup N_{\mathcal{G}_{2}}\right)=\emptyset$.

- Select some set $S_{1}$ of sinks from the copy of $\mathcal{G}_{1}$ in the sum $\mathcal{G}_{1}+\mathcal{G}_{2}$, and an equal-size set $S_{2}$ of sources from the copy of $\mathcal{G}_{2}$ in the sum.

- Pairwise identify (i.e., merge) the nodes in the sets $S_{1}$ and $S_{2}$ in some way. The resulting set of nodes is $\mathcal{G}$ 's node-set; the induced set of arcs is $\mathcal{G}$ 's arc-set. ${ }^{6}$

- Add the dag $\mathcal{G}$ thus obtained to the base set $\mathcal{B}$.

We denote the composition operation by $\Uparrow$ and say that $\mathcal{G}$ is composite of type $\left[\mathcal{G}_{1} \Uparrow \mathcal{G}_{2}\right]$.

The dag $\mathcal{G}$ is a $\triangleright$-linear composition of the CBBBs $\mathcal{G}_{1}, \ldots, \mathcal{G}_{n}$ if: $(a) \mathcal{G}$ is composite of type $\mathcal{G}_{1} \Uparrow \cdots \Uparrow \mathcal{G}_{n} ;(b)$ each $\mathcal{G}_{i} \triangleright \mathcal{G}_{i+1}$, for all $i \in[1, n-1]$.

The following results underlie our decomposition-based scheduling algorithm.

Lemma 2.1 ([16]). (a) If a schedule $\Sigma$ for a dag $\mathcal{G}$ is altered to execute all of $\mathcal{G}$ 's nonsinks before any of its sinks, then the IC quality of the resulting schedule is no less than $\Sigma$ 's. (b) The relation $\triangleright$ is transitive. (c) The composition operation is associative.

Theorem 2.1 ([16]). Let $\mathcal{G}$ be a $\triangleright$-linear composition of $\mathcal{G}_{1}, \ldots, \mathcal{G}_{n}$, where each $\mathcal{G}_{i}$ admits an IC-optimal schedule $\Sigma_{i}$. The schedule $\Sigma$ for $\mathcal{G}$ that proceeds as follows is IC optimal.

1. For $i=1, \ldots, n$, in turn, $\Sigma$ executes the nodes of $\mathcal{G}$ that correspond to nonsinks of $\mathcal{G}_{i}$, in the order mandated by $\Sigma_{i}$.

2. $\Sigma$ finally executes all sinks of $\mathcal{G}$ in any order.

\footnotetext{
${ }^{4}[a, b]$ denotes the set of integers $\{a, a+1, \ldots, b\}$.

${ }^{5}$ Continuing the practice of [16], our base dags here are CBBBs.

${ }^{6} \mathrm{An}$ arc $(u \rightarrow v)$ is induced if $\{u, v\} \subseteq N_{\mathcal{G}}$.
} 
One finds in [16] a suite of algorithms that determine whether or not a given dag $\mathcal{G}$ can be decomposed into a set of CBBBs $\left\{\mathcal{G}_{i}\right\}$ that satisfy Theorem 2.1; they compute an ICoptimal schedule for $\mathcal{G}$ whenever the theorem does apply. We summarize the algorithms to suggest their low-degree polynomial, yet superlinear complexity.

1. Remove from $\mathcal{G}$ all "shortcuts," i.e., arcs whose removal does not alter connectivity; cf. [11];

2. Parse $\mathcal{G}$, when possible, into $\mathrm{CBBB}$ sia a greedy algorithm;

3. Use the parsing to transform $\mathcal{G}$ into a "super-dag" of CBBBs whose arcs denote compositions;

4. Use a stable sorting algorithm [6] to determine if $\mathcal{G}$ is a $\triangleright$-linear composition of its CBBBs (so that Theorem 2.1 yields an IC-optimal schedule for $\mathcal{G}$ ).

The nascent scheduling theory of [16] is illustrated there via a small repertoire of CBBBs that lead, via Theorem 2.1, to a rich family of complex dags that we know how to schedule IC optimally (including the dags in Fig. 2). This early success motivates the challenge we address in Section 3: to expand the repertoire of CBBBs that the theory can handle. The complexity of the scheduling algorithms derived in [16] from Theorem 2.1 motivates the challenge we address in Section 4: to identify situations in which we can bypass those algorithms by having a dag "inherit" an IC-optimal algorithm from some kindred dag.

\section{Expanding the Repertoire of Building Blocks}

This section is devoted to expanding the repertoire of CBBBs that the scheduling algorithms of Section 2.3 have access to in [16]. We focus only on the problem of finding IC-optimal schedules for the CBBBs we consider, relying on the algorithm developed in [4] to find all possible $\triangle$-priorities among these dags.

\subsection{Planar Bipartite Trees}

We strive for an extensive repertoire of CBBBs: $(a)$ that compose into dags that one might encounter in real computations; $(b)$ that our theory shows how to schedule optimally and prioritize. Although our main focus is on dags that are either expansive - growing outward from their sources - or reductive - growing inward toward their sinks (cf. Figs. 2 and 4), we gain a technical advantage by considering also a CBBB that is a combination of expansive and reductive. Specifically, by demonstrating how to schedule any such combined CBBB IC optimally, we demonstrate in one fell swoop how to schedule IC optimally any CBBB that is either expansive or reductive. 
Planar Bipartite Trees. A sequence of positive numbers is zigzagged if it alternates integers and reciprocals of integers, with all integers exceeding 1 . For any zigzagged sequence $\hat{\delta}$, the $\hat{\delta}$-Planar Bipartite Tree (PBT, for short), denoted $\mathcal{P}[\hat{\delta}]$, is defined inductively as follows; see Fig. 4.

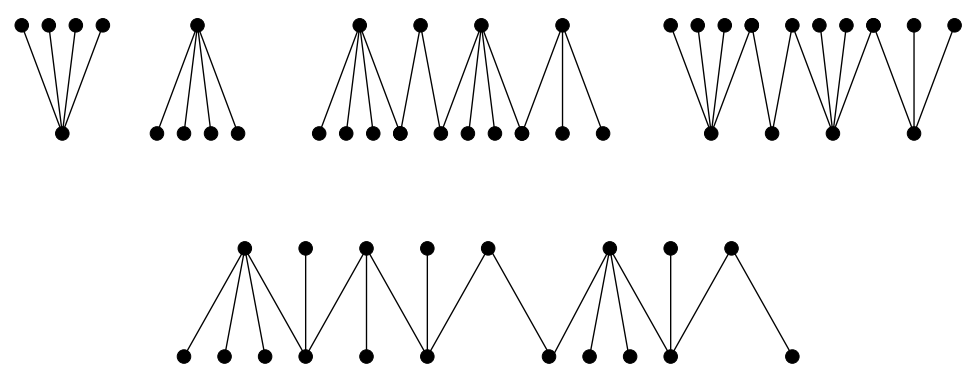

Figure 4: Top: $\mathcal{W}[4], \mathcal{M}[4], \mathcal{M}[4,2,4,3], \mathcal{W}[4,2,4,3] ;$ bottom: $\mathcal{P}\left[\frac{1}{4}, 3, \frac{1}{3}, 3, \frac{1}{2}, 2, \frac{1}{4}, 3, \frac{1}{2}\right]$. Edges represent arcs that point upward.

The base cases. For each $d>1$ :

- $\mathcal{P}[d]$ is the (single-source) outdegree- $d \mathbf{W}$-dag $\mathcal{W}[d]$, i.e., the bipartite dag that has one source, $d$ sinks, and $d$ arcs connecting the source to each sink.

- $\mathcal{P}\left[\frac{1}{d}\right]$ is the (single-sink) indegree-d M-dag $\mathcal{M}[d]$, i.e., the bipartite dag that has one sink, $d$ sources, and $d$ arcs connecting each source to the sink.

The inductive extensions. For each zigzagged sequence $\hat{\delta}$ and each $d>1$ :

- If $\hat{\delta}$ ends with a reciprocal, then $\mathcal{P}[\hat{\delta}, d]$ is obtained by giving $d-1$ new sinks to $\mathcal{P}[\hat{\delta}]$, with $\mathcal{P}[\hat{\delta}]$ 's rightmost source as their common parent.

- If $\hat{\delta}$ ends with an integer, then $\mathcal{P}\left[\hat{\delta}, \frac{1}{d}\right]$ is obtained by giving $d-1$ new sources to $\mathcal{P}[\hat{\delta}]$, with $\mathcal{P}[\hat{\delta}]$ 's rightmost sink as their common child.

Special PBTs. Two classes of PBTs deserve special mention. For any sequence of integers, $\hat{\delta}=d_{1}, d_{2}, \ldots, d_{k}$, each $>1$ : the $\hat{\delta}$-strand of $\mathbf{W}$-dags, denoted $\mathcal{W}[\hat{\delta}]$, and the $\hat{\delta}$-strand of M-dags, denoted $\mathcal{M}[\hat{\delta}]$ are defined as follows.

$$
\begin{aligned}
\mathcal{W}\left[d_{1}, d_{2}, \ldots, d_{k}\right] & =\mathcal{P}\left[d_{1}, \frac{1}{2}, d_{2}, \frac{1}{2}, \ldots, \frac{1}{2}, d_{k}\right] \\
\mathcal{M}\left[d_{1}, d_{2}, \ldots, d_{k}\right] & =\mathcal{P}\left[\frac{1}{d_{1}}, 2, \frac{1}{d_{2}}, 2, \ldots, 2, \frac{1}{d_{k}}\right] .
\end{aligned}
$$

We refer generically to $\mathcal{M}[\hat{\delta}]$ as an $\mathbf{M}$-strand and to $\mathcal{W}[\hat{\delta}]$ as a $\mathbf{W}$-strand. ${ }^{7}$ Note that every strand is connected, hence has no isolated nodes.

\footnotetext{
${ }^{7}$ In $[16], \mathrm{W}$-strands of the form $\mathcal{W}[2,2, \ldots, 2,1]$ are called $\mathbf{N}$-strands.
} 
Fig. 2 illustrates why we view W-strands and M-strands as the basic building blocks of expansive and reductive dags, respectively. The expansive mesh is composite of type $\mathcal{W}[2] \Uparrow$ $\mathcal{W}[2,2] \Uparrow \mathcal{W}[2,2,2] ;$ the expansive tree is composed of seven instances of $\mathcal{W}[2]$. Dually, the reductive mesh is composite of type $\mathcal{M}[2,2,2] \Uparrow \mathcal{M}[2,2] \Uparrow \mathcal{M}[2]$; the reductive tree is composed of seven instances of $\mathcal{M}[2]$. Fig. 5 illustrates this for the reductive mesh.

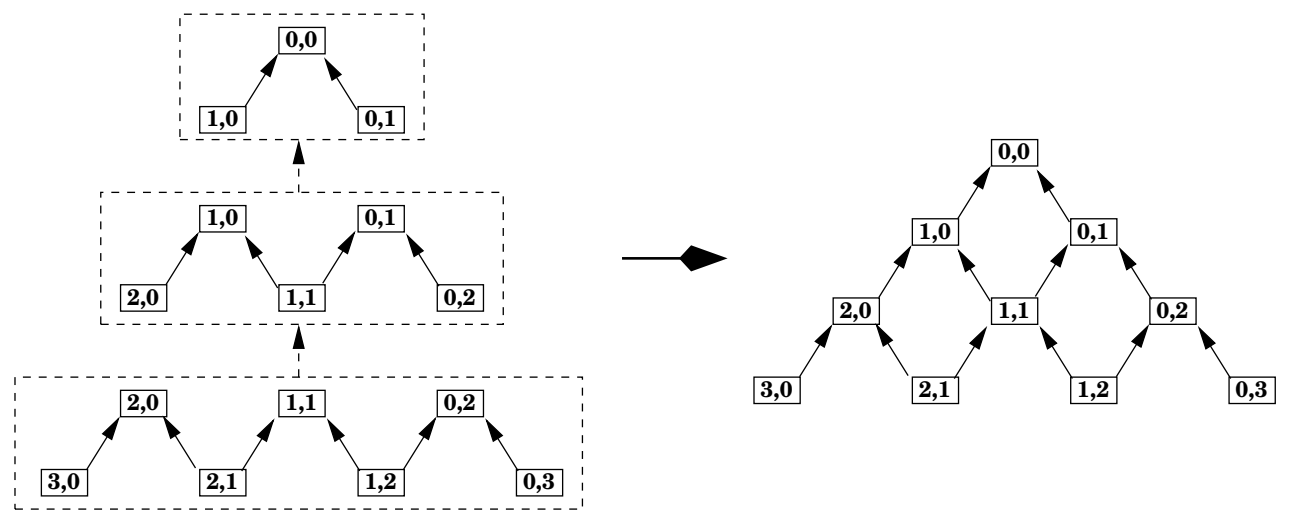

Figure 5: The reductive mesh of Fig. 2 is composite of type $\mathcal{M}[2,2,2] \Uparrow \mathcal{M}[2,2] \Uparrow \mathcal{M}[2]$.

\subsection{IC-Optimal Schedules for M- and W-Strands and PBTs}

Theorem 3.1. Every sum of PBTs admits an IC-optimal schedule.

Because M-strands and W-strands are special forms of PBTs, Theorem 3.1 immediately implies that every sum of $M$ - or of $W$-strands admits an IC-optimal schedule.

Our proof of Theorem 3.1 in fact proves a stronger assertion, which we develop now.

Focus on an arbitrary sum of PBTs $\mathcal{S}$. By Lemma 2.1(a), we may restrict attention to schedules that execute all of $\mathcal{S}$ 's sources before any of its sinks. Let $\operatorname{Src}(\mathcal{S})$ denote the set of $\mathcal{S}$ 's sources. For any $X \subseteq \operatorname{Src}(\mathcal{S})$, denote by $e(X ; \mathcal{S})$ the number of sinks of $\mathcal{S}$ that are ELIGIBLE at the step when the sources in $X$ are precisely those that have been executed.

Focus on a planar drawing of $\mathcal{S}$, strand by strand, so that we can identify its sources, from left to right, with the integers $1,2, \ldots, n$, where $n=|\operatorname{Src}(\mathcal{S})|$. For any $u \in \operatorname{Src}(\mathcal{S})$ and any $k \in[1, n]$, denote by $u[k]$ the set $u[k]=\{u, u+1, \ldots, u+k-1\}$ comprising source $u$ and the $k-1$ sources to its right in the drawing. To simplify exposition, we allow $k$ to exceed $n+1-u$, so that $u[k]$ may contain numbers greater than $n$-but none of these large integers denotes a node of $\mathcal{S}$. Let $\mathcal{S}_{u}$ denote the strand of $\mathcal{S}$ that $u$ belongs to, and let $S_{u}=\operatorname{Src}\left(\mathcal{S}_{u}\right)$. Denote by $e_{k}(u)$ the number $e_{k}(u)=e\left(u[k] \cap S_{u} ; \mathcal{S}\right)$ of sinks of $\mathcal{S}$ that are rendered ELIGIBLE when the sources in $u[k]$ are the only EXECUTED sources of $\mathcal{S}$. Associate 
with each $u \in \operatorname{Src}(\mathcal{S})$ the $n$-component eligiblity vector $V_{u}=\left\langle e_{1}(u), e_{2}(u), \ldots, e_{n}(u)\right\rangle$. Note that, since $|\operatorname{Src}(\mathcal{S})|=n$, if $u[k]$ contains numbers larger than $n$, then the last $u$ entries of $V_{u}$ are identical. Finally, order the vectors $V_{1}, V_{2}, \ldots, V_{n}$ in lexicographic order, using the notation $V_{v} \geq_{L} V_{w}$ to indicate that source $v$ 's vector precedes source $w$ 's vector lexicographically. We call a source $s \in \operatorname{Src}(\mathcal{S})$ maximal if $V_{s} \geq_{L} V_{s^{\prime}}$ for all $s^{\prime} \in \operatorname{Src}(\mathcal{S})$.

A schedule $\Sigma_{\mathcal{S}}$ for the sum of PBTs $\mathcal{S}$ is $\geq_{L^{-}}$greedy if it operates as follows.

1. $\Sigma_{\mathcal{S}}$ chooses any maximal $s \in \operatorname{Src}(\mathcal{S})$ as the first source of $\mathcal{S}$ to execute.

2. After executing source $s, \Sigma_{\mathcal{S}}$ removes from $\mathcal{S}$ source $s$ and all sinks (if any) that have $s$ as their only parent. This converts $\mathcal{S}$ to a new sum of PBTs $\mathcal{S}^{\prime}$ whose constituent strands are as follows.

(a) Each PBT of $\mathcal{S}$ other than $\mathcal{S}_{s}$ (i.e., each strand that does not contain $s$ ) is a constituent strand of $\mathcal{S}^{\prime}$

(b) $\mathcal{S}_{s}$ contributes to $\mathcal{S}^{\prime}$ all of its nodes other than $s$ and all sinks that have $s$ as their only parent.

The number of constituent strands of $\mathcal{S}^{\prime}$ is either one smaller than the number of constituent strands of $\mathcal{S}$ (if $s$ is $\mathcal{S}_{s}$ 's only source), or is the same as that number (if $s$ is either the leftmost or rightmost source of $\mathcal{S}_{s}$ ), or is one greater than that number (in all other cases).

3. $\Sigma_{\mathcal{S}}$ recursively executes the sum $\mathcal{S}^{\prime}$ using the $\geq_{L^{-}}$greedy schedule $\Sigma_{\mathcal{S}^{\prime}}$.

We prove Theorem 3.1 via the following more detailed result.

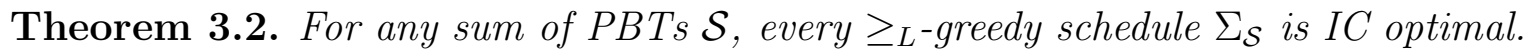

(Intuitively the "lookahead" inherent in eligiblity vectors prevents such a $\Sigma_{\mathcal{S}}$ from "getting stuck in" local optima that are not globally optimal.) We prove Theorem 3.2 by induction, in the next two subsections.

\subsection{1 $\Sigma_{\mathcal{S}}$ chooses the best first source to execute}

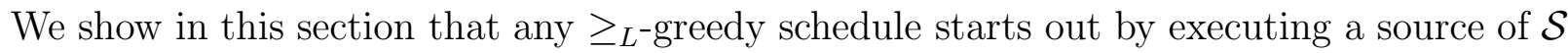
that is most advantageous (with respect to IC quality).

Lemma 3.1. Let $\mathcal{S}$ be an arbitrary sum of PBTs, and let $s$ be an arbitrary maximal source of $\mathcal{S}$. For each set $X \subseteq \operatorname{Src}(\mathcal{S})$, there is a set $X^{\prime} \subseteq \operatorname{Src}(\mathcal{S})$ of equal cardinality such that: (a) $s \in X^{\prime}$, and $(b) e\left(X^{\prime} ; \mathcal{S}\right) \geq e(X ; \mathcal{S})$. 
Proof. For any maximal source $s$ of $\mathcal{S}$, focus on an arbitrary $X \subseteq \operatorname{Src}(\mathcal{S})$ that does not contain $s$. Let $w \in X$ be any source and $\ell \in[1, n]$ any integer such that $w[\ell]$ is a maximal cardinality sequence of consecutive numbers from $X \cap S_{w}$ (using the integer names of sources). The maximality of source $s$ ensures that $V_{s} \geq_{L} V_{w}$; i.e., either $V_{s}=V_{w}$, or there exists $h \in[0, n-1]$ such that $s(1)=w(1), s(2)=w(2), \ldots, s(h)=w(h)$, and $s(h+1)>w(h+1)$.

We now investigate the impact on the number of ELIGIBLE sinks at step $|X|$ of the execution of $\mathcal{S}$, of "un-executing" certain sources from set $X$ and executing an equal number of other sources in their stead. Specifically, we "un-execute" some EXECUTED sources near to, and including $\mathrm{w}$, and execute an equal number of un-EXECUTED sources near to, and including s. (This substitution for certain nodes in $X$ will yield the set $X^{\prime}$.)

Focus on the following two quantities.

- $h^{\star} \in[0, n-1]$ is the largest value such that $e_{1}(s)=e_{1}(w), e_{2}(s)=e_{2}(w), \ldots, e_{h^{\star}}(s)=$ $e_{h^{\star}}(w)$. It is, thus, the maximum number of consecutive sources starting from $s$ whose execution does not improve on the "yield" produced by starting from $w$.

- $k^{\star} \in[1, \ell]$ is the largest value such that $s\left[k^{\star}\right] \cap X=\emptyset$. It is, thus, the maximum number of consecutive sources starting from $s$ that are not EXECUTED at the start of our modification of $X$.

We branch on the relative sizes of $h^{\star}$ and $k^{\star}$.

Case 1: $h^{\star}<k^{\star}$.

Consider the impact of "unexecuting" the sources in $w\left[h^{\star}+1\right]$ and executing, in their steads, the sources in $s\left[h^{\star}+1\right]$ (which, in this case, is a subset of $S_{s}$ ). This replaces the set $X$ by $X^{\prime}=\left(X \backslash w\left[h^{\star}+1\right]\right) \cup s\left[h^{\star}+1\right]$. The "unexecution" loses us at most $e_{h^{\star}+1}(w)+1$ ELIGIBLE sinks. (The possible extra sink may be lost if the rightmost source in $w\left[h^{\star}+1\right]$ has a righthand neighbor in $\mathcal{S}_{w} \cap X$ with which it shares a child.) In compensation, the execution gains us at least $e_{h^{\star}+1}(s) \geq e_{h^{\star}+1}(w)+1$ new ELIGIBLE sinks. We are, thus, not worse off for the exchange.

Case 2: $h^{\star} \geq k^{\star}$. We have two subcases to consider.

2a. $e_{k^{\star}}(w)=e_{\ell}(w)$.

In this case, we know two significant pieces of information.

1. Once having executed nodes $w, w+1, \ldots, w+k^{\star}-1$, no further sinks were rendered ELIGIBLE by executing any source in $\left\{w+k^{\star}, \ldots, w+\ell-1\right\}$. (Recall that $w[\ell] \subseteq X$.)

2. All of nodes $s, s+1, \ldots, s+k^{\star}-1$ belong to $S_{s}$, and none belongs to $X$.

This information tells us that "unexecuting" the sources in $w\left[k^{\star}\right]$ loses us exactly $e_{k^{\star}}(w)$ ELIGIBLE sinks, while executing the sources in $s\left[k^{\star}\right]$ gains us at least $s_{k^{\star}}(w)=e_{k^{\star}}(w)$ new 
ELIGIBLE sinks. This means that replacing the set $X$ by $X^{\prime}=\left(X \backslash w\left[k^{\star}\right]\right) \cup s\left[k^{\star}\right]$ leaves us with at least as many ELIGIBLE sinks. As in Case 1, we are not worse off for the exchange.

2b. $e_{k^{\star}}(w)<e_{\ell}(w)$.

In this case, even having executed nodes $w, w+1, \ldots, w+k^{\star}-1$, some new sinks are rendered ELIGIBLE by executing the sources in $\left\{w+k^{\star}, \ldots, w+\ell-1\right\}$. This means that $s+k^{\star} \in S_{s}$, i.e., that the integer $s+k^{\star}$ is the name of a real source of $\mathcal{S}$. To wit, were this not true, we would have $e_{k^{\star}+1}(s)=e_{k^{\star}}(s)$ for all $i>1$, so that, in particular, $e_{\ell}(s)<e_{\ell}(w)$. This would contradict the fact that $V_{s} \geq_{L} V_{w}$.

Let $t$ be the leftmost child of $s+k^{\star}$ (using the planar drawing of $\mathcal{S}$ to specify "leftmost"). Since source $s+k^{\star}-1 \notin X, t$ was not rendered ELIGIBLE by the execution of the sources in $X$. Further, since $s+k^{\star} \notin s\left[k^{\star}\right], t$ will not be rendered ELIGIBLE by the execution of the sources in $s\left[k^{\star}\right]$.

We digress to introduce a useful notion. A source (resp., sink) $u$ of a PBT is a backbone source (resp., sink) if $u$ is either extremal in the strand (i.e., leftmost or rightmost), or $u$ has at least two children (resp., at least two parents). We note the obvious, yet important, fact that every maximal source is a backbone source. To wit, at least one of the two neighboring (in the planar drawing) backbone sources of each non-backbone source $s$ has a lexicographically larger eligibility vector than $s$.

Our analysis now branches on whether or not $s+k^{\star}$ is a backbone source of $\mathcal{S}$.

$\mathbf{2 b}(\mathbf{i})$. If $s+k^{\star}$ is a backbone source, then we know four significant facts.

1. $s+k^{\star} \in X$ because $h^{\star} \geq k^{\star}$

2. $s+k^{\star}$ is a backbone source by assumption;

3. $s$ is a backbone source because it is maximal;

4. $s+k^{\star} \notin w\left[k^{\star}\right] \quad$ because $V_{s} \geq_{L} V_{w}$ and $h^{\star} \geq k^{\star}$.

It follows that executing the nodes in $X \cup s\left[k^{*}\right]$ renders sink $t$ ELIGIBLE. Therefore, "unexecuting" the sources in $w\left[k^{\star}\right]$ loses us at most $e_{k^{\star}}(w)+1$ ELIGIBLE sinks. In compensation, executing the sources in $s\left[k^{\star}\right]$ gains us at least $e_{k^{\star}}(s)+1$ ELIGIBLE sinks-because $t$ becomes ELIGIBLE when source $s+k^{\star}-1$ is executed. Thus, replacing $X$ with $X^{\prime}=\left(X \backslash w\left[k^{\star}\right]\right) \cup s\left[k^{\star}\right]$ leaves us with at least as many ELIGIBLE sinks.

$\mathbf{2 b}$ (ii). If $s+k^{\star}$ is not a backbone source, then in order to render sink $t$ ELIGIBLE, we must execute not only $s+k^{\star}$, but also the set $R \subset S_{s}$ that comprises all of the sources to $s+k^{\star}$ 's right (in the drawing), until we reach the next backbone source.

Let $j^{\star} \in[0, n]$ be the largest integer such that $e_{k^{\star}}(w)=e_{k^{\star}+j^{\star}}(w)$; i.e., $e_{k^{\star}}(w)=e_{k^{\star}+1}(w)=$ $e_{k^{\star}+2}(w)=e_{k^{\star}+j^{\star}}(w)<e_{k^{\star}+j^{\star}+1}(w)$. Since the current case is defined by the condition " $e_{k^{\star}}(w)<e_{\ell}(w)$," we know that every element of $w\left[k^{\star}+j^{\star}\right]$ belongs to $X$.

Easily, $|R| \leq j^{\star}$. Were this not the case, then we would have $e_{k^{\star}+j^{\star}+1}(s)=e_{k^{\star}+j^{\star}}(s)=$ $\cdots=e_{k^{\star}}(s)=e_{k^{\star}}(w)<e_{k^{\star}+j^{\star}+1}(w)$. This would contradict the fact that $V_{s} \geq_{L} V_{w}$. 
Note now that "unexecuting" the $k^{\star}+j^{\star}$ sources in $w\left[k^{\star}+j^{\star}\right]$ would lose us no more than $e_{k^{\star}}(w)+1$ ELIGIBLE sinks. In compensation, executing the $k^{\star}$ sources in $s\left[k^{\star}\right]$, plus the at-most $j^{\star}$ sources in $R$ gains us at least $e_{k^{\star}}(s)+1$ ELIGIBLE sinks (because sink $t$ becomes ELIGIBLE). Thus, replacing the set $X$ with the (possibly smaller) set $X^{\prime}=$ $\left(X \backslash w\left[k^{\star}+j^{\star}\right]\right) \cup\left(s\left[k^{\star}\right] \cup R\right)$ leaves us with at least as many ELIGIBLE sinks.

The sequences of cases we have considered have established the following. By executing a sequence of sources starting with $s$, instead of a like-numbered sequence starting with $w$, we can only increase the total number of ELIGIBLE sinks. The lemma follows.

\subsection{2 $\Sigma_{\mathcal{S}}$ continues to make good choices}

Assume, for induction, that every $\geq_{L}$-greedy schedule is IC optimal for all sums of PBTs having $n$ or fewer sources. Lemma 3.1 verifies the $(n=1)$ case of this assertion. Focus, therefore, on an arbitrary sums of PBTs having $n+1$ sources. Lemma 3.1 shows that every $\geq_{L}$-greedy schedule, $\Sigma_{\mathcal{S}}$, chooses an optimal source $s \in \operatorname{Src}(\mathcal{S})$ to execute in its first step. Hence, if we seek a set $X \subseteq \operatorname{Src}(\mathcal{S})$ that renders maximally many sinks of $\mathcal{S}$ ELIGIBLE, among cardinality- $|X|$ subsets of $\operatorname{Src}(\mathcal{S})$, we can, with no loss of generality, choose $X$ to contain $s$. Let $\mathcal{S}^{\prime}$ be the sum of PBTs obtained by removing from $\mathcal{S}$ source $s$ plus all sinks that have $s$ as their only parent.

Let the yield, $Y l d(v)$, of a source $v$ be the number of sinks that are rendered ELIGIBLE by executing just $v$. Removing $s$ and its single-parent children effectively increases by 1 the yields of the sources that neighbor $s$ in $\mathcal{S}_{s}$, if any.

Easily $e(X ; \mathcal{S})=Y l d(s)+e\left(X \backslash\{s\} ; \mathcal{S}^{\prime}\right)$. Since $\mathcal{S}^{\prime}$ has fewer sources than $\mathcal{S}$, our inductive assumption asserts that schedule $\Sigma_{\mathcal{S}^{\prime}}$ is IC optimal for $\mathcal{S}^{\prime}$. It follows that schedule $\Sigma_{\mathcal{S}}$, which executes $s$ and then mimics schedule $\Sigma_{\mathcal{S}^{\prime}}$, is IC optimal for $\mathcal{S}$.

This completes the proof of Theorem 3.2, hence, also, of Theorem 3.1.

\section{Exploiting Duality when Scheduling Dags}

The results in this section apply to arbitrary dags, not just bipartite ones. Indeed, these results represent a significant step in liberating our scheduling theory from its focus on dags that are obtained via composition from any particular repertoire of CBBBs.

The dual of a $\operatorname{dag} \mathcal{G}$ is the $\operatorname{dag} \widetilde{\mathcal{G}}$ that is obtained by reversing all of $\widetilde{\mathcal{G}}^{\prime}$ 's arcs. Clearly, the sources of $\mathcal{G}$ are the sinks of $\widetilde{\mathcal{G}}$, and the sinks of $\mathcal{G}$ are the sources of $\widetilde{\mathcal{G}}$.

Note that the dual of a PBT is another PBT; the dual of an M-strand is a Wstrand; the dual of a W-strand is an M-strand. Specifically, for all alternations 
of integers and reciprocals, $\widehat{\mathcal{P}}\left[d_{1}, d_{2}, \ldots, d_{n}\right]=\mathcal{P}\left[\frac{1}{d_{1}}, \frac{1}{d_{2}}, \ldots, \frac{1}{d_{n}}\right]$.

In this section, we demonstrate that, for any $\operatorname{dag} \mathcal{G}$ :

- one can easily "read off" an IC-optimal schedule for either of $\mathcal{G}$ or $\widetilde{\mathcal{G}}$ from an ICoptimal schedule for the other (Section 4.1);

- one can easily determine $\triangleright$-priority relationships of either $\mathcal{G}$ or $\widetilde{\mathcal{G}}$ from $\triangleright$-priority relationships of the other (Section 4.2).

Although the results in this section do not depend in any way on the composite-dag framework of Theorem 2.1 (and the resulting scheduling algorithms of [16]), they do imply that the theorem always applies to an arbitrary $\operatorname{dag} \mathcal{G}$ and its dual, $\widetilde{\mathcal{G}}$, simultaneously. (See Corollary 5.1 for a formal verification.)

\subsection{Scheduling-Based Duality}

Let $\mathcal{G}$ be a dag that has $n$ nonsinks, comprising the set $U=\left\{u_{1}, u_{2}, \ldots, u_{n}\right\}$, and $N$ nonsourcess, comprising the set $\left\{v_{1}, v_{2}, \ldots, v_{N}\right\}$. Let $\Sigma$ be a schedule for $\mathcal{G}$ that executes $\mathcal{G}$ 's nonsinks in the order

$$
u_{k_{1}}, u_{k_{2}}, \ldots, u_{k_{n-1}}, u_{k_{n}}
$$

(after which, $\Sigma$ executes all of $\mathcal{G}$ 's sinks). Each node-execution of a nonsink, say $u_{k_{j}}$, renders ELIGIBLE a (possibly empty) "packet" of nonsources, $P_{j}=\left\{v_{j, 1}, \ldots, v_{j, i_{j}}\right\}$ of $\mathcal{G}$. (Clearly, $\mathcal{G}$ must have an arc from $u_{k_{j}}$ to each node in $P_{j}$.) Thus, $\Sigma$ renders $\mathcal{G}$ 's nonsources ELIGIBLE in a sequence of such "packets:"

$$
P_{1}=\left\{v_{1,1}, \ldots, v_{1, i_{1}}\right\}, P_{2}=\left\{v_{2,1}, \ldots, v_{2, i_{2}}\right\}, \ldots, P_{n}=\left\{v_{n, 1}, \ldots, v_{n, i_{n}}\right\} .
$$

A schedule $\widetilde{\Sigma}$ for $\mathcal{G}$ 's dual dag, $\widetilde{\mathcal{G}}$, is dual to $\Sigma$ if it executes $\widetilde{\mathcal{G}}$ 's nonsinks - which, recall, are $\mathcal{G}$ 's nonsources - in an order of the form

$$
\left[\left[v_{n, 1}, \ldots, v_{n, i_{n}}\right]\right],\left[\left[v_{n-1,1}, \ldots, v_{n-1, i_{n-1}}\right]\right], \ldots,\left[\left[v_{1,1}, \ldots, v_{1, i_{1}}\right]\right]
$$

where $[[a, b, \ldots, c]]$ denotes a fixed, but unspecified, permutation of the set $\{a, b, \ldots, c\}$ (after which, $\widetilde{\Sigma}$ executes all of $\widetilde{\mathcal{G}}$ 's sinks). Note that $\widetilde{\mathcal{G}}$ will generally admit many schedules that are dual to $\Sigma$. Note also that both $\Sigma$ and $\widetilde{\Sigma}$ honor Lemma 2.1(a).

Theorem 4.1. Let the dag $\mathcal{G}$ admit the IC-optimal schedule $\Sigma_{\mathcal{G}}$. Any schedule for $\widetilde{\mathcal{G}}$ that is dual to $\Sigma_{\mathcal{G}}$ is IC optimal. 
Proof. Let $\Sigma_{\mathcal{G}}$ execute $\mathcal{G}$ 's nonsinks in the order (2) and render $\mathcal{G}$ 's nonsources ELIGIBLE according to the partial order specified implicitly by (3). It follows that every schedule for $\widetilde{\mathcal{G}}$ that is dual to $\Sigma_{\mathcal{G}}$ executes $\widetilde{\mathcal{G}}$ 's $N$ nonsinks in an order of the form (4).

Assume now, for contradiction, that some schedule for $\widetilde{\mathcal{G}}$, call it, $\Sigma_{\widetilde{\mathcal{G}}}$, is dual to $\Sigma_{\mathcal{G}}$ but is not IC optimal. There must then be a schedule $\Sigma_{\widetilde{\mathcal{G}}}^{\prime}$ for $\widetilde{\mathcal{G}}$ and a step $t \in[1, N-1]$ such that $E_{\Sigma_{\widetilde{\mathcal{G}}}^{\prime}}(t)>E_{\Sigma_{\widetilde{\mathcal{G}}}}(t)$. We show now that the existence of schedule $\Sigma_{\widetilde{\mathcal{G}}}^{\prime}$ refutes the alleged IC optimality of schedule $\Sigma_{\mathcal{G}}$.

Let $s \in[1, n]$ be the smallest number of nonsinks of $\mathcal{G}$ that schedule $\Sigma_{\mathcal{G}}$ needs to execute in order to render $\geq N-t$ nonsources of $\mathcal{G}$ ELIGIBLE. In other words, $E_{\Sigma_{\mathcal{G}}}(s) \geq N-t$, but $E_{\Sigma_{\mathcal{G}}}\left(s^{\prime}\right)<N-t$ for all $s^{\prime}<s$. We claim that

$$
E_{\Sigma_{\tilde{\mathcal{G}}}}(t) \geq n-s
$$

To verify this inequality, note that, as $\Sigma_{\mathcal{G}}$ executes nonsinks $u_{k_{1}}, u_{k_{2}}, \ldots, u_{k_{s}}$ of $\mathcal{G}$ during its first $s$ steps, it renders ELIGIBLE the nonsources of $\mathcal{G}$ in the set $P_{1} \cup P_{2} \cup \cdots \cup P_{s}$; cf. (2, $3)$. Since $E_{\Sigma_{\mathcal{G}}}(s) \geq N-t$, it follows that $t^{\prime} \stackrel{\text { def }}{=}\left|P_{s+1} \cup P_{s+2} \cup \cdots \cup P_{n}\right| \leq t$.

Since $\Sigma_{\widetilde{\mathcal{G}}}$ is dual to $\Sigma_{\mathcal{G}}$, during its first $t^{\prime}$ steps, $\Sigma_{\widetilde{\mathcal{G}}}$ executes precisely the $t^{\prime}$ nonsinks of $\widetilde{\mathcal{G}}$ in the set $P_{s+1} \cup P_{s+2} \cup \cdots \cup P_{n}$. Because schedule $\Sigma_{\mathcal{G}}$ is IC optimal, each node in $\left\{u_{k_{1}}, u_{k_{2}}, \ldots, u_{k_{s}}\right\}$ must have -in $\mathcal{G}$ - an arc to one or more nodes in $P_{1} \cup P_{2} \cup \cdots \cup P_{s}$. Were this not the case, $\Sigma_{\mathcal{G}}$ could render $\geq N-t$ sinks of $\mathcal{G}$ ELIGIBLE by executing (at most) $s-1$ nonsinks of $\mathcal{G}$. It thus follows that the set of nonsources of $\widetilde{\mathcal{G}}$ that are rendered ELIGIBLE by $\Sigma_{\widetilde{\mathcal{G}}}$ 's first $t^{\prime}$ node-executions is precisely the set $\left\{u_{k_{s+1}}, u_{k_{s+2}}, \ldots, u_{k_{n}}\right\}$, so that $E_{\Sigma_{\widetilde{\mathcal{G}}}}\left(t^{\prime}\right)=n-s$. Since $t \geq t^{\prime}$, this verifies inequality $(5)$.

Recall now that, by hypothesis, $E_{\Sigma_{\tilde{\mathcal{G}}}^{\prime}}(t)>E_{\Sigma_{\tilde{\mathcal{G}}}}(t)$. There must, then, be a set $V=$ $\left\{v_{1}, v_{2}, \ldots, v_{t}\right\}$ of $t$ nonsinks of $\widetilde{\mathcal{G}}$ that schedule $\Sigma_{\widetilde{\mathcal{G}}}^{\prime}$ executes during its first $t$ steps, thereby rendering ELIGIBLE a set $S$ comprising at least $n-s+1$ nonsources of $\widetilde{\mathcal{G}}$. Clearly, there is no arc in $\widetilde{\mathcal{G}}$ from any node of $\bar{V}$ to any node of $S$. It follows that any schedule $\Sigma_{\mathcal{G}}^{\prime}$ for $\mathcal{G}$ that executes the $s^{\prime} \leq s-1$ nonsinks of $\mathcal{G}$ in the set $\bar{S}$ during its first $s^{\prime}$ steps renders ELIGIBLE all of the nonsources of $\mathcal{G}$ in the set $\bar{V}$. But these nonsources are at least $N-t$ in number! It follows that $E_{\Sigma_{\mathcal{G}}^{\prime}}(s-1)>E_{\Sigma_{\mathcal{G}}}(s-1)$, which contradicts the alleged IC optimality of schedule $\Sigma_{\mathcal{G}}$.

We conclude that schedule $\Sigma_{\widetilde{\mathcal{G}}}^{\prime}$ cannot exist, whence the (arbitrary dual) schedule $\Sigma_{\widetilde{\mathcal{G}}}$ is IC optimal for $\widetilde{\mathcal{G}}$, as was claimed.

The following corollary of Theorem 4.1 is immediate from the fact that $\widetilde{\widetilde{G}}=\mathcal{G}$.

Corollary 4.1. A dag $\mathcal{G}$ admits an IC-optimal schedule if, and only if, its dual, $\widetilde{\mathcal{G}}$, does. 


\subsection{Priority-Based Duality}

We now derive $\triangleright$-priorities between two dags, $\mathcal{G}_{1}$ and $\mathcal{G}_{2}$, from $\triangleright$-priorities between their dual dags, $\widetilde{\mathcal{G}}_{1}$ and $\widetilde{\mathcal{G}}_{2}$, respectively. For this purpose, it is convenient to supplement the system of inequalities (1) that defines $\triangleright$-priority with a dual formulation of the relation.

Let $\mathcal{G}$ be a dag that has $N$ nonsources, and let $\Sigma$ be a schedule for $\mathcal{G}$. For any $e \in[0, N]$, let $X_{\Sigma}(e)$ be the smallest number of nonsinks of $\mathcal{G}$ that must be executed in order to render at least $e$ nonsources of $\mathcal{G}$ ELigiBLE. Let $\mathcal{G}_{1}$ and $\mathcal{G}_{2}$ be disjoint dags that, respectively, have $N_{1}$ and $N_{2}$ nonsources and admit IC-optimal schedules $\Sigma_{1}$ and $\Sigma_{2}$. If the following inequalities hold:

$$
\begin{aligned}
& \left(\forall e \in\left[0, N_{1}\right]\right)\left(\forall f \in\left[0, N_{2}\right]\right): \\
& X_{\Sigma_{1}}(e)+X_{\Sigma_{2}}(f) \geq X_{\Sigma_{1}}\left(\min \left\{e+f, N_{1}\right\}\right)+X_{\Sigma_{2}}\left(\max \left\{0, e+f-N_{1}\right\}\right),
\end{aligned}
$$

then $\mathcal{G}_{1}$ has dual priority over $\mathcal{G}_{2}$, denoted $\mathcal{G}_{1} \widetilde{\triangleright} \mathcal{G}_{2}$. Clearly, the relations $\widetilde{\triangleright}$ and $\triangleright$ are equivalent: the former relation strives to minimize the number of EXECUTED nonsinks for a given number of ELIGIBLE nonsources; the latter strives to maximize the number of ELIGIBLE nonsources for a given number of EXECUTED nonsinks. Stated formally,

Lemma 4.1. If the dags $\mathcal{G}_{1}$ and $\mathcal{G}_{2}$ admit IC-optimal schedules $\Sigma_{1}$ and $\Sigma_{2}$, respectively, then $\mathcal{G}_{1} \triangleright \mathcal{G}_{2}$ if, and only if, $\mathcal{G}_{1} \widetilde{\triangleright} \mathcal{G}_{2}$.

Lemma 4.1 affords us easy access to the following result. (Note the reversal of indices.)

Theorem 4.2. For all dags $\mathcal{G}_{1}$ and $\mathcal{G}_{2}: \mathcal{G}_{1} \triangleright \mathcal{G}_{2}$ if, and only if, $\widetilde{\mathcal{G}}_{2} \triangleright \widetilde{\mathcal{G}}_{1}$.

Proof. For $i=1,2$, let $\mathcal{G}_{i}$ have $n_{i}$ nonsinks and $N_{i}$ nonsources, and let it admit the ICoptimal schedule $\Sigma_{i}$. Theorem 4.1 tells us how to construct, from $\Sigma_{i}$ an IC-optimal schedule $\widetilde{\Sigma}_{i}$ for $\widetilde{\mathcal{G}}_{i}$. Moreover, the proof of that theorem gives us valuable information about how $\Sigma_{i}$ and $\widetilde{\Sigma}_{i}$ operate. Specifically (using the notation of the proof), recall that, in order to render $N-j$ nonsources of $\widetilde{\mathcal{G}}$ ELIGIBLE, we must execute $j$ nonsinks in packets $P_{j+1}, P_{j+2}, \ldots, P_{N}-$ or, equivalently, in order to render $j$ nonsources of $\widetilde{\mathcal{G}}$ ELIGIBLE, we must execute $j$ nonsinks in packets $P_{N-j+1}, P_{N-j+2}, \ldots, P_{N}$. Moreover, executing $j$ nonsinks of $\mathcal{G}$ renders ELIGIBLE exactly the nonsources of packets $P_{1}, P_{2}, \ldots, P_{j}$. Hence, for all $e \in\left[0, n_{i}\right]$,

$$
\begin{aligned}
X_{\widetilde{\Sigma}_{i}}(e) & =\left|P_{n_{i}-e+1} \cup P_{n_{i}-e+2} \cup \cdots \cup P_{n_{i}}\right| \\
& =N_{i}-\left|P_{1} \cup P_{2} \cup \cdots \cup P_{n_{i}-e}\right| \\
& =N_{i}-E_{\Sigma_{i}}\left(n_{i}-e\right) .
\end{aligned}
$$

Thus, for any $e \in\left[0, n_{1}\right]$ and $f \in\left[0, n_{2}\right]$, we have

$$
X_{\widetilde{\Sigma}_{1}}(e)+X_{\widetilde{\Sigma}_{2}}(f)=\left(N_{1}+N_{2}\right)-E_{\Sigma_{1}}\left(n_{1}-e\right)-E_{\Sigma_{2}}\left(n_{2}-f\right) .
$$


Say now that $\mathcal{G}_{1} \triangleright \mathcal{G}_{2}$. By system (1), we then have

$$
E_{\Sigma_{1}}\left(n_{1}-e\right)+E_{\Sigma_{2}}\left(n_{2}-f\right) \leq E_{\Sigma_{1}}\left(\min \left\{n_{1}, n_{1}+n_{2}-e-f\right\}\right)+E_{\Sigma_{2}}\left(\max \left\{0, n_{2}-e-f\right\}\right) .
$$

Combining this inequality with (7), we find that

$$
\begin{aligned}
X_{\widetilde{\Sigma}_{1}}(e)+X_{\widetilde{\Sigma}_{2}}(f) \geq & \left(N_{1}+N_{2}\right)-E_{\Sigma_{1}}\left(\min \left\{n_{1}, n_{1}+n_{2}-e-f\right\}\right) \\
& -E_{\Sigma_{2}}\left(\max \left\{0, n_{2}-e-f\right\}\right) \\
=\quad X_{\widetilde{\Sigma}_{1}} & \left(n_{1}-\min \left\{n_{1}, n_{1}+n_{2}-e-f\right\}\right) \\
& \quad+X_{\widetilde{\Sigma}_{2}}\left(n_{2}-\max \left\{0, n_{2}-e-f\right\}\right) \\
= & X_{\widetilde{\Sigma}_{1}}\left(\max \left\{0, e+f-n_{2}\right\}\right)+X_{\widetilde{\Sigma}_{2}}\left(\min \left\{n_{2}, e+f\right\}\right) .
\end{aligned}
$$

This last inequality means that $\widetilde{\mathcal{G}}_{2} \widetilde{\triangleright} \widetilde{\mathcal{G}}_{1}$, so that, by Lemma $4.1, \widetilde{\mathcal{G}}_{2} \triangleright \widetilde{\mathcal{G}}_{1}$, as claimed. The converse follows "by running the argument backwards."

\section{Where We Are, and Where We're Going}

\subsection{Conclusions}

The results in this paper significantly expand, via two avenues, the range of dags that the algorithmic theory of [16] can schedule IC optimally.

Expanding the repertoire of building blocks. In addition to the structurally uniform dags of Fig. 2 and the simple composite dags of [16], we are now able to schedule IC optimally any sequence of sums of PBTs that is linearly ordered under $\triangleright$-priority: $\mathcal{P}_{1} \triangleright$ $\mathcal{P}_{2} \triangleright \cdots \triangleright \mathcal{P}_{n}$. As but one significant illustration, we are now able to schedule IC optimally the following three classes of dags, which are reminiscent of the dags encountered in a variety of scientific computations. Let us be given:

- any sequence of sums of $W$-strands that is linearly ordered under $\triangleright$-priority: $\breve{\mathcal{S}}_{1} \triangleright$ $\breve{\mathcal{S}}_{2} \triangleright \cdots \triangleright \breve{\mathcal{S}}_{n}$

- $\underset{\widetilde{\mathcal{S}}}{\text { any sequence of }}$ sums of $M$-strands that is linearly ordered under $\triangleright$-priority: $\widetilde{\mathcal{S}}_{1} \triangleright$ $\widetilde{\mathcal{S}}_{2} \triangleright \cdots \triangleright \widetilde{\mathcal{S}}_{m}$

Then any composite dag of one of the following three types admits the IC-optimal schedule dictated by Theorem 2.1:
(1) $\breve{\mathcal{S}}_{1} \Uparrow \cdots \Uparrow \breve{\mathcal{S}}_{n}$,
(2) $\widetilde{\mathcal{S}}_{1} \Uparrow \cdots \Uparrow \widetilde{\mathcal{S}}_{m}$,
(3) $\breve{\mathcal{S}}_{1} \Uparrow \cdots \Uparrow \breve{\mathcal{S}}_{n} \Uparrow \widetilde{\mathcal{S}}_{1} \Uparrow \cdots \Uparrow \widetilde{\mathcal{S}}_{m}$. 
Informally, we now have scheduling control over a broad family of dags that are expansive, reductive, and expansive-reductive (in the way that, e.g., many series-parallel [17] or forkjoin dags are). Fig. 6 exhibits two simple dags (constructed from those in Fig. 4) that exemplify the expanded repertoire of dags that we can schedule IC optimally because of our expanded repertoire of building blocks. In the figure: the lefthand dag is composite of type $\mathcal{W}[4] \Uparrow \mathcal{W}[4,2,4,3] \Uparrow \mathcal{M}[4,2,4,3] \Uparrow \mathcal{M}[4]$, and the righthand dag is composite of type $\mathcal{W}[5] \Uparrow \widetilde{\mathcal{P}}\left[\frac{1}{4}, 3, \frac{1}{3}, 3, \frac{1}{2}, 2, \frac{1}{4}, 3, \frac{1}{2}\right] \Uparrow \mathcal{P}\left[\frac{1}{4}, 3, \frac{1}{3}, 3, \frac{1}{2}, 2, \frac{1}{4}, 3, \frac{1}{2}\right] \Uparrow \mathcal{M}[6] ;$ straightforward calculations using (1) show that $\mathcal{W}[4] \triangleright \mathcal{W}[4,2,4,3] \triangleright \mathcal{M}[4,2,4,3] \triangleright \mathcal{M}[4]$ and that $\mathcal{W}[5] \triangleright$ $\widetilde{\mathcal{P}}\left[\frac{1}{4}, 3, \frac{1}{3}, 3, \frac{1}{2}, 2, \frac{1}{4}, 3, \frac{1}{2}\right] \triangleright \mathcal{P}\left[\frac{1}{4}, 3, \frac{1}{3}, 3, \frac{1}{2}, 2, \frac{1}{4}, 3, \frac{1}{2}\right] \triangleright \mathcal{M}[6]$.

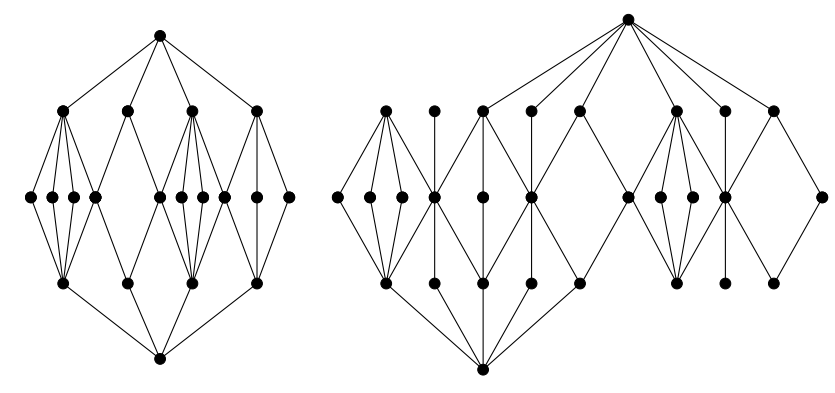

Figure 6: Two dags that can now be scheduled IC optimally. Edges represent arcs that point upward.

Exploiting duality to schedule dags optimally. We have shown how to "read off" an IC-optimal schedule for an arbitrary $\operatorname{dag} \mathcal{G}$ from an IC-optimal schedule for $\mathcal{G}$ 's dual. We have also shown how to "read off" $\triangleright$-priorities among a collection of dags from $\triangleright$-priorities among their dual dags. This allows one to shortcut the algorithmic suite from [16] and its upcoming sequel [4] when dealing with dags whose duals have previously been dealt with. Alternatively, one can actually incorporate duality into the algorithmic suite, as suggested by the following corollary to Theorems 4.1 and 4.2 .

Corollary 5.1. If the dag $\mathcal{G}$ admits an IC-optimal schedule via Theorem 2.1, then so also does its dual dag $\widetilde{\mathcal{G}}$.

Proof. The premise of the corollary implies that the dag $\mathcal{G}$ is composite of type $\mathcal{G}_{1} \Uparrow$ $\mathcal{G}_{2} \Uparrow \cdots \Uparrow \mathcal{G}_{n}$ for some bipartite $\operatorname{dags} \mathcal{G}_{1}, \mathcal{G}_{2}, \ldots, \mathcal{G}_{n}$ that admit IC-optimal schedules, $\Sigma_{1}, \Sigma_{2}, \ldots, \Sigma_{n}$, respectively, and that form a linear chain of $\triangleright$-priorities, $\mathcal{G}_{1} \triangleright \mathcal{G}_{2} \triangleright \cdots \triangleright$ $\mathcal{G}_{n}$. Consider the bipartite dags $\widetilde{\mathcal{G}}_{1}, \widetilde{\mathcal{G}}_{2}, \ldots, \widetilde{\mathcal{G}}_{n}$ that are, respectively, dual to the dags $\mathcal{G}_{1}, \mathcal{G}_{2}, \ldots, \mathcal{G}_{n}$.

We note first that the $\operatorname{dag} \widetilde{\mathcal{G}}$ that is dual to $\mathcal{G}$ is composite of type $\widetilde{\mathcal{G}}_{n} \Uparrow \widetilde{\mathcal{G}}_{n-1} \Uparrow \ldots \Uparrow \widetilde{\mathcal{G}}_{1}$. We leave the verification of this easy fact to the reader.

Next, by Theorem 4.1, we know that, for each $i \in[1, n]$, the dag $\widetilde{\mathcal{G}}_{i}$ admits an IC-optimal schedule - specifically, one that is dual to the IC-optimal schedule $\Sigma_{i}$ of $\mathcal{G}_{i}$. 
Finally, by Theorem 4.2 , the dags $\widetilde{\mathcal{G}}_{1}, \widetilde{\mathcal{G}}_{2}, \ldots, \widetilde{\mathcal{G}}_{n}$ form a linear chain of $\triangleright$-priorities, $\widetilde{\mathcal{G}}_{n} \triangleright$ $\widetilde{\mathcal{G}}_{n-1} \triangleright \cdots \triangleright \widetilde{\mathcal{G}}_{1}$.

In summation, $\mathcal{G}$ 's dual dag $\widetilde{\mathcal{G}}$ satisfies the conditions of Theorem 2.1 whenever $\mathcal{G}$ does, hence admits an IC-optimal schedule via the formula of that theorem.

\subsection{Projections}

Our work on this project proceeds in several directions.

Theory. We are engaged in investigations aimed at extending the scope of the theory of [16] in a number of ways.

1. We are expanding the scheduling component of our theory to move further beyond its current dependence on $\triangleright$-linear compositions of building blocks. Our new work focuses on (IC-optimal) schedules that allow the interleaved execution of building blocks. Preliminary results appear in [4].

2. We are seeking a rigorous framework for devising schedules that are "approximately" IC optimal. This thrust is important for computational reasons - a computationally simple heuristic may be "almost as good" as a more arduously derived IC-optimal schedule - and because many dags do not admit IC-optimal schedules.

3. We are working to extend our theory so that it can optimally schedule composite dags whose building blocks are not necessarily bipartite.

Simulations and experiments. We are engaged in a suite of simulation experiments that seek to determine the extent to which our scheduling algorithms actually enhance the efficiency of Internet-based computations. A report on simulations involving real scientific dags appears in [14]; a report on artificially generated ones is being prepared [10].

Integration with grid schedulers. Our most ambitious non-theoretical endeavor involves incorporating our suite of scheduling algorithms into a real scheduling tool, the Condor DAGMan tool [3]. Coauthor G. Malewicz developed a tool [14] for prioritizing the jobs of a DAGMan file, while visiting Argonne National Lab.

Acknowledgments. A portion of the research of G. Cordasco and G. Malewicz was done while visiting the Univ. of Massachusetts Amherst. A portion of the research of G. Malewicz was done while visiting the Mathematics and Computer Science Division of Argonne National Lab. The research of G. Malewicz was supported in part by NSF Grant ITR-800864. The research of A. Rosenberg was supported in part by NSF Grant CCF0342417. The authors are grateful to Ian Foster and Michael Wilde (Argonne) and to Matt Yurkewych (NSA) for valuable conversations at several stages of the research reported here. 


\section{References}

[1] R. Buyya, D. Abramson, J. Giddy (2001): A case for economy Grid architecture for service oriented Grid computing. 10th Heterogeneous Computing Wkshp.

[2] W. Cirne and K. Marzullo (1999): The Computational Co-Op: gathering clusters into a metacomputer. 13th Intl. Parallel Processing Symp., 160-166.

[3] Condor Project, University of Wisconsin. http://www.cs.wisc.edu/condor

[4] G. Cordasco, G. Malewicz, A.L. Rosenberg (2006): Extending IC-scheduling theory via the sweep algorithm. Typescript, Univ. Massachusetts; submitted for publication.

[5] G. Cordasco, G. Malewicz, A.L. Rosenberg (2007): Applying IC-scheduling theory to familiar classes of computations. Wkshp. on Large-Scale, Volatile Desktop Grids (PCGrid'O'7) (2007).

[6] T.H. Cormen, C.E. Leiserson, R.L. Rivest, C. Stein (1999): Introduction to Algorithms (2nd Edition). MIT Press, Cambridge, Mass.

[7] I. Foster and C. Kesselman [eds.] (2004): The Grid: Blueprint for a New Computing Infrastructure (2nd Edition). Morgan-Kaufmann, San Francisco.

[8] I. Foster, C. Kesselman, S. Tuecke (2001): The anatomy of the Grid: enabling scalable virtual organizations. Intl. J. Supercomputer Applications.

[9] L. Gao and G. Malewicz (2006): Toward maximizing the quality of results of dependent tasks computed unreliably. Theory of Computing Systs., to appear. See also, Intl. Conf. on Principles of Distributed Systems, 2004.

[10] R. Hall, A.L. Rosenberg, A. Venkataramani (2007): A comparison of dag-scheduling strategies for Internet-based computing. Intl. Parallel and Distributed Processing Symp.

[11] H.T. Hsu (1975): An algorithm for finding a minimal equivalent graph of a digraph. J. ACM 22, 11-16.

[12] D. Kondo, H. Casanova, E. Wing, F. Berman (2002): Models and scheduling mechanisms for global computing applications. Intl. Parallel and Distr. Processing Symp.

[13] E. Korpela, D. Werthimer, D. Anderson, J. Cobb, M. Lebofsky (2000): SETI@home: massively distributed computing for SETI. In Computing in Science and Engineering (P.F. Dubois, Ed.) IEEE Computer Soc. Press, Los Alamitos, CA. 
[14] G. Malewicz, I. Foster, A.L. Rosenberg, M. Wilde (2007): A tool for prioritizing DAGMan jobs and its evaluation." J. Grid Computing, to appear.

[15] G. Malewicz and A.L. Rosenberg (2005): On batch-scheduling dags for Internet-based computing. Euro-Par 2005. In Lecture Notes in Computer Science 3648, SpringerVerlag, Berlin, 262-271.

[16] G. Malewicz, A.L. Rosenberg, M. Yurkewych (2006): Toward a theory for scheduling dags in Internet-based computing. IEEE Trans. Comput. 55, 757-768.

[17] M. Mitchell (2004): Creating minimal vertex series parallel graphs from directed acyclic graphs. Australasian Symp. on Information Visualisation. In Conferences in Research and Practice in Information Technology 35 (N. Churcher and C. Churcher, Eds.) ACS Press, pp. 133-139.

[18] A.L. Rosenberg (2004): On scheduling mesh-structured computations for Internetbased computing. IEEE Trans. Comput. 53, 1176-1186.

[19] A.L. Rosenberg and M. Yurkewych (2005): Guidelines for scheduling some common computation-dags for Internet-based computing. IEEE Trans. Comput. 54, 428-438.

[20] X.-H. Sun and M. Wu (2003): GHS: A performance prediction and node scheduling system for Grid computing. IEEE Intl. Parallel and Distributed Processing Symp. 